\title{
Turbulent momentum transport in core tokamak plasmas and penetration of scrape-off layer flows
}

\author{
J. Abiteboul $\ddagger$, Ph. Ghendrih, V. Grandgirard, \\ T. Cartier-Michaud, G. Dif-Pradalier, X. Garbet, G. Latu, \\ C. Passeron, Y. Sarazin, A. Strugarek, O. Thomine and \\ D. Zarzoso
}

CEA, IRFM, F-13108 Saint-Paul-lez-Durance, France.

E-mail: jeremie.abiteboul@gmail.com

\begin{abstract}
The turbulent transport of toroidal angular momentum in the core of a tokamak plasma is investigated in global, full- $f$ gyrokinetic simulations, performed with the GYSELA code in the flux-driven regime. During the initial turbulent phase, a front of positive Reynolds stress propagates radially, generating intrinsic toroidal rotation from a vanishing initial profile. This is also accompanied by a propagating front of turbulent heat flux. In the statistical steady-state regime, turbulent transport exhibits large-scale avalanche-like events which are found to transport both heat and momentum, and similar statistical properties are obtained for both fluxes. The impact of scrape-off layer flows is also investigated by modifying the boundary conditions in the simulations. The observed impact is radially localized for L-mode like poloidal profiles of parallel velocity at the edge, while a constant velocity at the edge can modify the core toroidal rotation profile in a large fraction of the radial domain.
\end{abstract}

PACS numbers: 52.35.Ra,52.30.-q,52.30.Gz,52.55.Fa,52.40.Hf

‡ Present address: Max-Planck-Institut für Plasmaphysik, Boltzmannstr. 2, D-85748 Garching, Germany 


\section{Introduction}

Toroidal rotation is generally understood to be beneficial for the performance of tokamaks, as it tends to stabilize the so-called resistive wall modes [1] and may also contribute, in the case of a sheared rotation profile, to the saturation of turbulent transport. In most present tokamaks, toroidal rotation is largely controlled externally thanks to Neutral Beam Injection (NBI). In ITER however, although the torque from NBI will be larger than in present tokamaks, the large moment of inertia of the plasma implies that this torque is not expected to lead to significant toroidal rotation velocities. Thus, there has recently been much interest in the generation of toroidal rotation by intrinsic mechanisms and in the transport of toroidal angular momentum by the plasma,

In the absence of any external torque, a number of different mechanisms can drive toroidal rotation. Generally speaking, any breaking of the axisymmetry in a tokamak can lead to the generation of toroidal rotation. This symmetry can be broken by a non-axisymmetric magnetic field, which acts trough collisional processes as a friction term on the toroidal velocity (see for example $[2,3,4]$ ). For instance, modifications of the toroidal rotation profile have been reported experimentally in the presence of toroidal field ripple $[5,6,7]$ or externally applied magnetic perturbations $[8,9]$. Another means of breaking the axisymmetry, which is the focus of the present work, is through electrostatic turbulence, which generates a non-axisymmetric electric potential.

Turbulent transport of toroidal momentum is investigated here in the specific case of electrostatic ion temperature gradient (ITG) driven turbulence, which is relevant for the tokamak core. In this limit, turbulent processes can be modelled using the gyrokinetic equation for the distribution of ion gyrocenters coupled to the quasi-neutrality equation to compute the electrostatic potential. A review of recent progress in gyrokinetic simulations can be found in [10]. Recently, several gyrokinetic codes have been developed to perform simulations in the flux-driven regime, i.e. forcing the turbulence by a prescribed heat source $[11,12,13]$, in order to resemble the experimental forcing.

Including a heat source in the plasma core and a sink near the edge allows one to model rather accurately the situation in experiments with regards to heat transport. Conversely, the question of boundary conditions is more complex in the context of momentum transport, and is crucial because of the local conservation of toroidal angular momentum, as verified by the gyrokinetic model $[14,15,16]$. The question is also relevant in experiments as the impact of scrape-off layer (SOL) flows on core toroidal rotation remains largely an open issue $[17,18,19]$. In the present work, this issue is investigated by considering various boundary conditions for toroidal rotation in gyrokinetic simulations. The code is run either with a constant - vanishing or finite - velocity or with a poloidal profile of parallel velocity imposed at the edge of the simulation domain.

In this paper we present new results concerning the statistical analysis of turbulent momentum transport in gyrokinetic simulations and the essential role of boundary conditions. The organization of this paper is as follows. The model used for the 
simulations with the GySELA code[20] is reviewed in section 2. The dynamics of turbulent heat and momentum transport are analyzed in section 3, both during the initial relaxation event observed in gyrokinetic simulations and in the steady-state of the saturated turbulent regime. In order to investigate the effect of edge flows on core rotation, the impact of boundary conditions on toroidal rotation is studied in section 4 .

\section{Flux-driven simulations with the gyrokinetic code GYSELA}

The simulations are performed with the global flux-driven gyrokinetic code GYSELA, which models the time evolution of the gyrocenter distribution $\bar{F}$, with no separation between equilibrium and perturbations. The semi-Lagrangian numerical scheme is used, as detailed in [21]. We consider the electrostatic limit and the low plasma $\beta$ limit, where $\beta$ is the ratio of the kinetic energy to the magnetic energy. The time evolution of $\bar{F}$ is given by the gyrokinetic equation in its conservative form [22], which reads

$$
\partial_{t} \bar{F}+\frac{1}{B_{\|}^{*}} \nabla_{\mathbf{z}} \cdot\left(\dot{\mathbf{z}} B_{\|}^{*} \bar{F}\right)=\mathcal{C}(\bar{F})+S,
$$

where we define $B_{\|}^{*}=B+\left(m v_{G \|} / e\right) \mathbf{b} \cdot(\nabla \times \mathbf{b})$, which corresponds to the volume element in guiding-center velocity space. The system of coordinates is $\mathbf{z}=\left(r, \theta, \varphi, v_{G \|}, \mu\right)$ where $r$ is the minor radius, $\theta$ is the poloidal angle, $\varphi$ is the toroidal angle, $v_{G \|}$ is the parallel velocity of the gyrocenter and $\mu$ is the adiabatic invariant. The equations of motion in (1) are the following

$$
\begin{aligned}
& B_{\|}^{*} d_{t} \mathbf{x}_{G}=v_{G \|} \mathbf{B}^{*}+\frac{1}{e} \mathbf{b} \times \nabla \Lambda, \\
& B_{\|}^{*} m d_{t} v_{G \|}=-\mathbf{B}^{*} \cdot \nabla \Lambda,
\end{aligned}
$$

where we define

$$
\begin{aligned}
\Lambda & =e \bar{\phi}+\mu B, \\
\mathbf{B}^{*} & =\mathbf{B}+\left(m v_{G \|} / e\right) \nabla \times \mathbf{b},
\end{aligned}
$$

where $m$ and $e$ are the species mass and charge, and $\bar{\phi}$ is the gyro-averaged electric potential. Self-consistency is obtained by coupling the gyrokinetic equation (1) with the quasi-neutrality constraint, which relates the electric potential to the distribution function. In the electrostatic limit, assuming a single ion species and adiabatic electron response, this equation reads

$$
-\nabla \cdot\left\{\frac{n_{e q} m}{B^{2}} \nabla_{\perp} \phi\right\}+\frac{n_{e q} e}{T}\left(\phi-\langle\phi\rangle_{F . S .}\right)=e \int 2 \pi B_{\|}^{*} \mathrm{~d} \mu \mathrm{d} v_{G \|} J \cdot\left(\bar{F}-\bar{F}_{e q}\right)(6)
$$

where $\bar{F}_{e q}$ is the equilibrium gyrocenter distribution function, associated with a vanishing electric potential and $\langle\phi\rangle_{F . S}$. is the flux-surface averaged electric potential. The gyroaverage operator $J$ is computed using a Padé approximation.

The right-hand side of (1) contains the collision operator $\mathcal{C}(\bar{F})$, which is implemented im GYSELA as a Fokker-Planck type operator acting on $v_{G \|}$ only. This operator conserves density, momentum and energy, and it was demonstrated that it 
allows one to recover the main results of neoclassical theory, in particular concerning the predictions for heat transport and poloidal rotation, including their dependence on aspect ratio and collisionality [23]. Additionally, the source term $S$ provides a constant input of heat in the system. This source is located near the inner radial boundary of the simulation domain and does not inject any particle or parallel momentum [24]. The heat sink is provided by an ad hoc diffusion term added to the right-hand side of (1) and non-vanishing only in a narrow radial region near the radial boundary of the simulation domain [12]. Using this source, flux-driven simulations can be performed where the temperature is fixed at the outer radial boundary and may evolve freely elsewhere. In this type of simulation, since the full distribution is evolved self-consistently with no assumption on the size of the perturbations, back-reactions of small-scale turbulence on the large-scale equilibrium are accounted for and the system may evolve far from its initial state. In terms of flows, boundary conditions impose a vanishing gradient at the inner radial boundary and zero parallel velocity at the outer boundary, corresponding to so-called no-slip boundary conditions. The role of these conditions in the simulations will be discussed further in the following.

\section{Dynamics of turbulent heat and momentum transport}

Using the gyrokinetic model in the flux-driven regime, the dynamics of both heat and momentum transport can be analyzed in fully self-consistent simulations. We consider here a simulation where the normalized gyroradius at the magnetic axis is $\rho_{*}=\rho_{i} / a=1 / 512$, where $\rho_{i}$ is the thermal Larmor radius and $a$ is the minor radius of the tokamak. This value is of the order of the expected $\rho_{*}$ in ITER. The normalized collisionality is $\nu^{*}=0.1$, corresponding to the so-called banana regime of neoclassical theory. The grid in $5 \mathrm{D}$ phase-space required for this simulation is $\left(N_{r}, N_{\theta}, N_{\varphi}, N_{v_{G \|}}, N_{\mu}\right)=(1024,1024,128,128,16)$, corresponding to approximately $310^{11}$ grid points. The safety factor profile is chosen as $q(r)=1+2.78(r / a)^{2.8}$ while the aspect ratio is $R_{0} / a=3.2$, where $R_{0}$ is the major radius at the magnetic axis. Since the adiabatic electron response impedes particle transport, the particle density profile remains constant, throughout the simulation. The density profile has a uniform logarithmic derivative in the simulation domain, $R_{0} / L_{n}=2$.

For flux-driven simulations, the ion temperature profile is allowed to evolve freely, and depends strongly on the amplitude of the source. In the statistical steadystate reached by this simulation, a mean ion temperature profile is obtained with $R_{0} / L_{T} \simeq 11.5$, where $L_{T}=-\left(d_{r} \log T\right)^{-1}$ is the temperature gradient length. With these paremeters, the plasma is well above the nonlinear threshold of the ITG instability. In simulations with lower ion temperature gradient, i.e. closer to the threshold, one may expect additional features to appear as the system approaches marginal stability, in particular in the form of time-independent zonal flow structures, as observed for instance in [25]. Thus, in such cases, the statistical analysis presented here would still be applicable but further work may be required in order to obtain a complete description 
of the complex self-organization processes at work. The dimensionless parameters used in the present simulations can be clarified by expressing them in physical units. For instance, considering a plasma with a major radius $R_{0}=2.2 \mathrm{~m}$, density $n_{0}=2.10^{19} \mathrm{~m}^{-3}$ and temperature $T_{0}=1 \mathrm{keV}$ at mid-radius, and with a magnetic field $B_{0}=2 \mathrm{~T}$ at the magnetic axis, one finds that the additional power corresponding to the source used here is approximately $P_{a d d} \simeq 0.6 \mathrm{MW} . \oint$

When initializing the system with a vanishing profile of toroidal rotation, it was previously reported [15] that, during the exponential growth phase of turbulence, a dipolar structure of toroidal rotation is generated by a large turbulent burst. This structure is consistent with the conservation of gyrocenter toroidal angular momentum [14, 15, 26], which has been shown to be recovered in gyrokinetic simulations [15]. A more detailed analysis reveals that this initial relaxation event is in fact characterized by an outward propagating front of both radial heat flux and Reynolds stress. Both quantities are represented in figure 1(a), at a given time during the propagation of the front. The time evolution is such that the two fronts, as well as the dipolar structure of toroidal rotation, are simultaneously propagating towards increasing minor radius. More precisely, one finds at each time that the center of the dipole (i.e., $\partial_{t} \mathcal{L}_{\varphi}=0$ where $\mathcal{L}_{\varphi}$ is the toroidal angular momentum), coincides with the maximum value of the Reynolds stress, consistently with the local equation for toroidal angular momentum transport [15]. As we are considering radial transport of toroidal momentum, the Reynolds stress considered here corresponds to the off-diagonal $(r, \phi)$ component. Toroidal momentum transport is often split into three terms: a diffusive contribution, a convective (or pinch) term [27, 28] and the so-called residual stress [29, 30]. For the event described here, as the initial profile of toroidal rotation is vanishing, the observed momentum flux corresponds only to a residual contribution. Note that, while the different contributions may be directly obtained in the steady-state regime from $\delta f$ simulations with specific equilibria [31], this splitting is not straightforward in a full- $f$ simulation as no scale separation is assumed between equilibrium and perturbations.

In order to clarify the dynamics of the propagation of heat and momentum fronts, a clearer picture can be obtained by representing the Reynolds stress as a function of the heat flux, for all radii and at a given time, as shown in figure 1(b). Note that a similar picture can be obtained by representing the quantities at a fixed radius and for increasing time, the choice made here is simply due to the greater resolution of the simulation diagnostics in the radial direction. With this representation, one can clearly identify a cycle, as both fluxes are vanishing at the inner and outer radial boundaries. An important result is, as can also be observed in figure 1(a), that the largest value of the Reynolds stress propagates ahead (i.e., is located further out radially at a given time) of the maximum heat flux. More precisely, the delay between the two maxima in figure 1 is of approximately $6 \rho_{i}$. The propagation velocity of the front can also be

$\S$ These figures are only provided as an example to illustrate the results: since the simulation only employs dimensionless parameters, other sets of parameters can also be considered for the same simulation. 


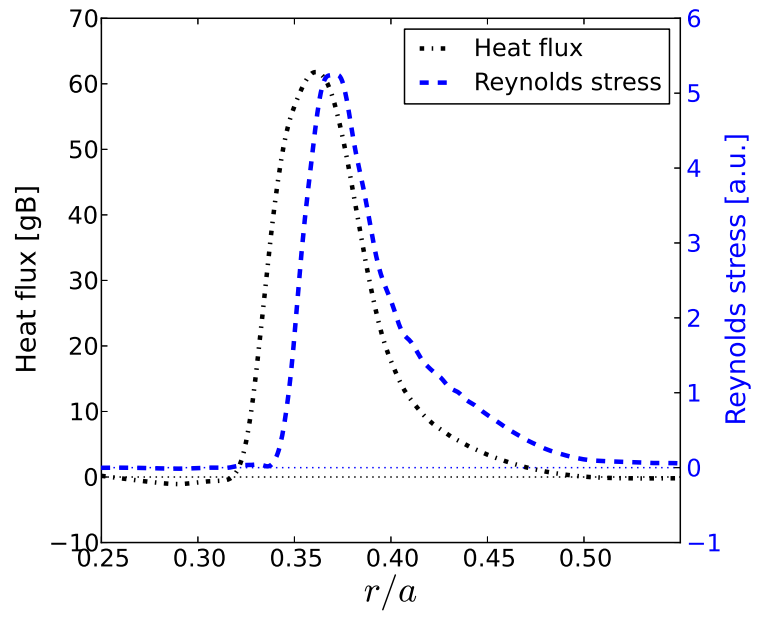

(a)

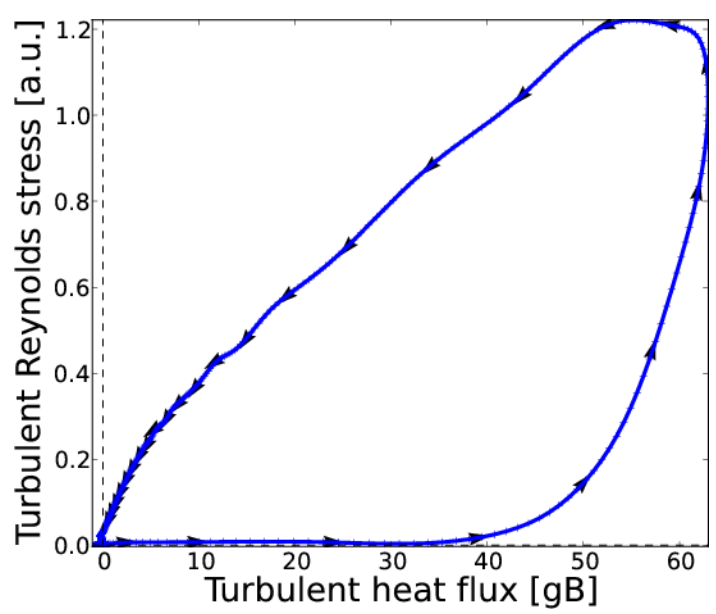

(b)

Figure 1. Propagation of a front of turbulent heat flux and generation of toroidal rotation: (a) Radial profiles of the heat flux and toroidal Reynolds stress at a given time during the initial turbulent burst. (b) Representation of the front (at the same simulation time) as a cycle in heat flux and Reynolds stress. The cycle starts and ends with both fluxes at zero, arrows represent the direction of increasing radius.

estimated by following the maximum of either flux over time, and is found to be of roughly five times the diamagnetic velocity, i.e. $V_{\text {front }} \simeq 5 \rho_{*} v_{T}$ where $v_{T}$ is the ion thermal velocity. Thus, one can compute the time delay between the maxima of heat flux and Reynolds stress, leading to approximately $600 \omega_{c}^{-1}$, which is of the order of the correlation time of turbulence $\tau_{c} \sim a / v_{T}$.

As previously mentioned, the gyrokinetic model ensures the conservation of toroidal momentum. Thus, in the absence of momentum fluxes at the boundaries, one would only obtain dipolar-like structures, with no net generation of toroidal rotation. In the simulation considered here, no-slip boundary conditions (i.e. $V_{\|}=0$ ) are imposed at the edge of the simulation domain. With no-slip boundary conditions, non-vanishing edge fluxes are possible, and therefore a net generation of toroidal rotation can occur. This occurs after the propagation of the initial turbulent front, when turbulence has spread to the edge regions of the simulation domain, where spatial diffusion is applied. During this phase of the simulation, a net profile of toroidal rotation builds up with a dominant contribution in the co-current direction (see figure 4 in [32]).

Eventually, a steady-state is reached with the saturation of turbulence, and we focus now on the dynamics of turbulent transport in this regime. For large simulations such as the one considered here, with $\rho_{*}=1 / 512$, the numerical cost of the simulations (in this case, approximately $6.10^{6} \mathrm{CPU}$ hours running on 8192 processors) implies that they cannot be run on confinement time scales. Thus, while turbulence has indeed reached a steady-state, the mean flows are still slowly evolving.

It was shown in [32], from simulations with the GYSELA and XGC1p [33] gyrokinetic 
codes, that both the turbulent heat flux and toroidal Reynolds stress exhibit largescale avalanche-like events. We recall that these avalanches are observed to propagate predominantly outward, although inward propagating fronts can also be observed. The fronts observed in the steady-state regime are found to propagate at velocities slightly lower than the diamagnetic velocity, i.e. $V_{\text {front }} \lesssim \rho_{*} v_{T}$ where $v_{T}$ is the ion thermal velocity.

The observations in [32] suggest that the same avalanches transport both heat and momentum. This result can indeed be highlighted by tracking the maxima of the turbulent heat flux and toroidal Reynolds stress during the steady-state of the simulation, as shown in figure 2(a). This confirms the observation in [32], as the maxima of the fluxes - which correspond to the large-scale avalanches - appear strongly correlated. Moreover, a delay can be identified between both fluxes, with the heat flux generally propagating ahead, in contrast with the observations made during the initial turbulent front, figure 1.

A representation of individual avalanches as cycles cannot be confirmed in the steady-state regime as the time evolution of the fluxes is less coherent and an accurate tracking of avalanche propagation is not straightforward. However, the link between avalanches of heat flux and momentum, clearly identified in figure 2(a), can be further quantified by analyzing the radial distance between the maxima of the fluxes in terms of statistical distributions, as presented in figure 2(b). First of all, the statistical distribution of the distance between two successive maxima of the heat flux is described by the dashed blue curve (labeled Q-Q). Although this distance exhibits significant variations, one can identify a strong peak corresponding to a typical distance of approximately 10 Larmor radii, which can also be translated in terms of transit times as roughly $2 a / c_{s}$, given the mean propagation velocity of the avalanches. We stress here that, as the fluxes considered here are flux-surface averaged, two successive avalanches may in fact be occuring at different poloidal or toroidal locations. Also, a secondary peak is observed, corresponding to the edges of the simulation domain, where the turbulent dynamics are not dominated by large-scale avalanches but rather by steady-state spatial structures of approximately 20 Larmor radii.

Second, the black curve (labeled R-Q) represents the distance between a heat flux maximum and the preceding Reynolds stress maximum. In most cases, the maximum of Reynolds stress is propagating with a slight delay behind the heat flux maximum, of the order of a Larmor radius. This statistical result clearly confirms the visual observation from figure 2(a). Finally, the solid blue curve (labeled Q-R) corresponds to the distance between a heat flux maximum and the following Reynolds stress maximum. By first considering the strongest peak in the statistical distribution, near $\Delta_{\rho}=10 \rho_{i}$, the time period between two avalanches and the delay between Reynolds stress and heat flux front, both previously obtained from the other distributions, are recovered. However, a significant number of events is also identified where the Reynolds stress is propagating ahead of the heat flux, as was the case for the initial turbulent front. The present data does not allow us to conclude as to what governs the relative positions of the two 


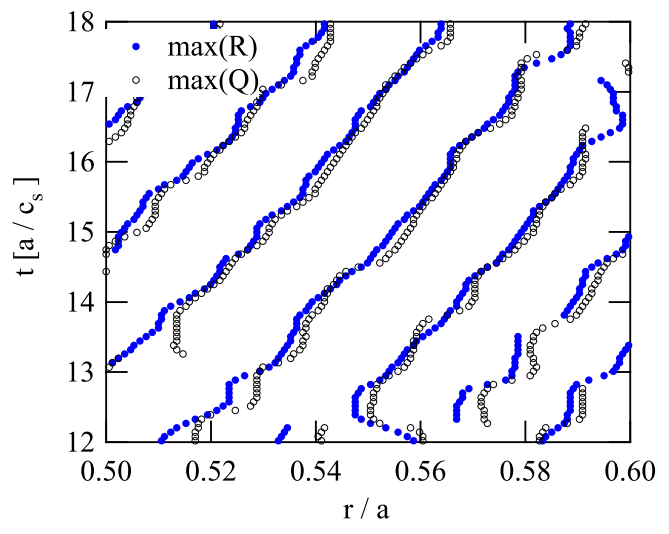

(a)

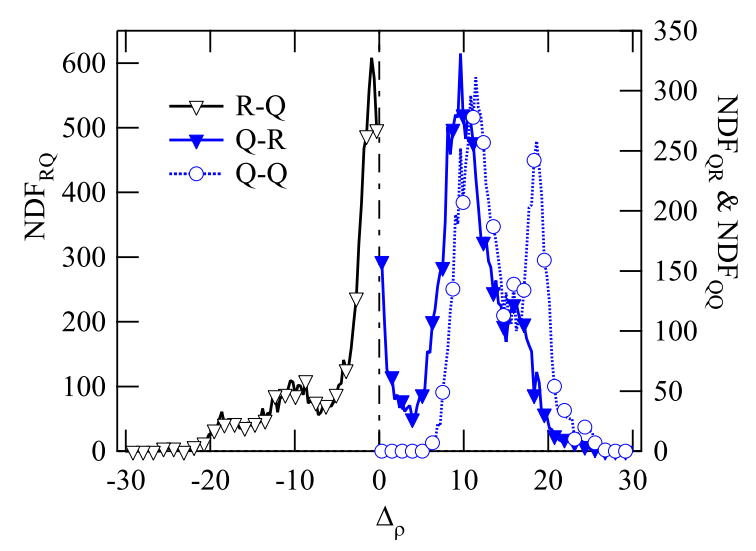

(b)

Figure 2. Avalanches of heat and momentum in the steady-state regime: (a) Spacetime representation of the maxima of turbulent heat flux (Q) and Reynolds stress (R). (b) Statistical distributions of the distances, in units of Larmor radius, between successive maxima of (black) Reynolds stress and heat flux, (solid blue) heat flux and Reynolds stress and (dashed blue) between two successive heat flux maxima.)

fronts, which do not appear to be related to the radial position of the avalanche. As the generation of toroidal Reynolds stress is necessarily linked to a breaking of symmetry, the relative position of the avalanches could be determined by the mechanism responsible for this symmetry breaking. Two key mechanisms have been identified in global gyrokinetic simulations, namely radial electric field shear and turbulence intensity gradient [34, 32]. However, no clear correlation is observed between the relative positions of the maxima and the importance of either mechanism. Another related open issue concerns the difference between the steady-state avalanches and the initial turbulent front, which propagates at a higher velocity and with opposite relative positions of the heat flux and Reynolds stress maxima. In order to resolve these issues, future work will focus on the relation between heat flux and Reynolds stress avalanches when varying the level of the turbulent drive - i.e. the distance from the nonlinear threshold of the instability - as well as the nature of the underlying instability.

In summary, the simple picture one can obtain from figure $2(\mathrm{~b})$ is of quasi-periodic avalanches, with a mean delay of $2 a / c_{s}$ between them, while for most of the avalanches the toroidal Reynolds stress front propagates with a delay of the order of $0.2 a / c_{s}$ relative to the heat flux front.

In this regime of statistical steady-state, heat and momentum transport can also be compared by analyzing the probability distribution functions (PDFs) of the turbulent heat flux and toroidal Reynolds stress. Basic comparisons between the XGC1p and GYSELA code were previously reported in [32], here we present a more detailed analysis of these statistics. We compute the PDFs of the flux-surface averaged fluxes for a radial 


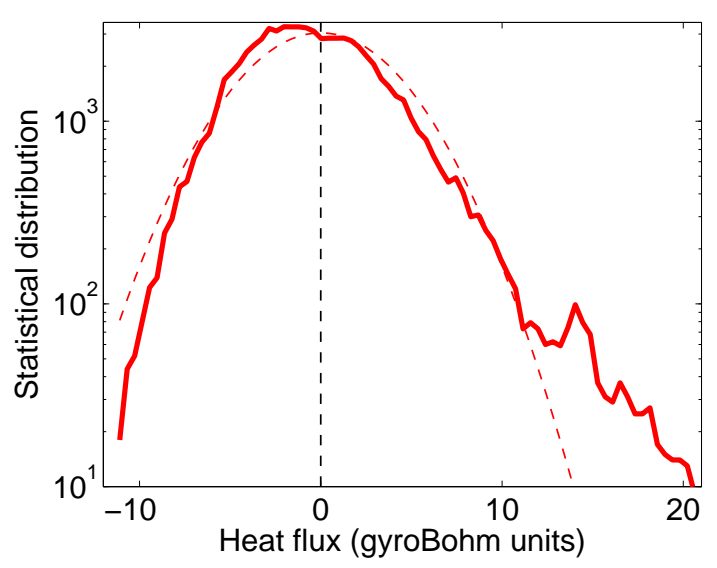

(a)

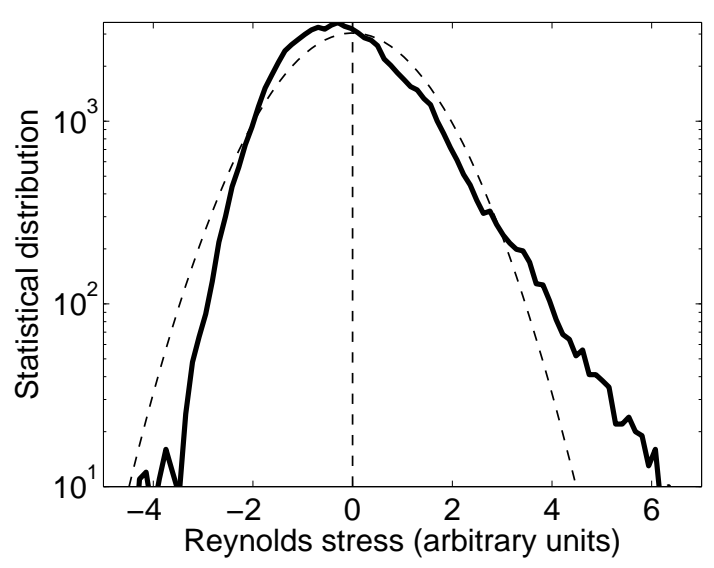

(b)

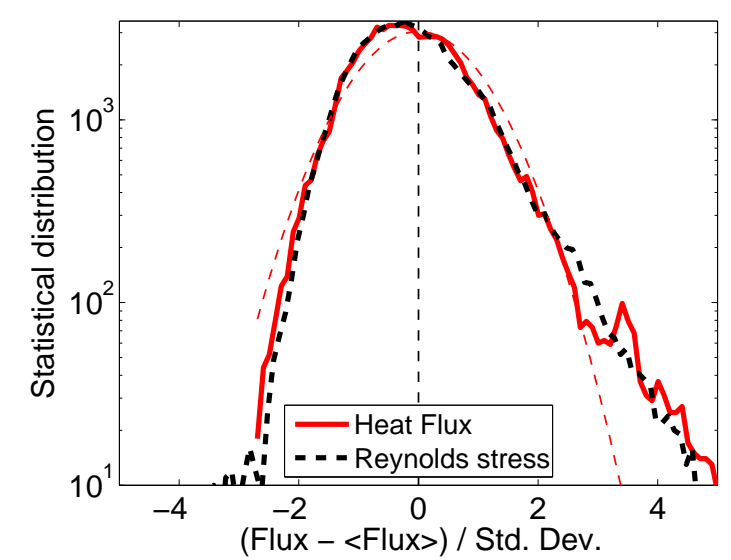

(c)

Figure 3. Statistical distribution functions of the turbulent (a) heat flux and (b) toroidal $(r, \varphi)$ Reynolds stress, around mid-radius $\mathrm{r} / \mathrm{a}=0.5$. In both cases, with a Gaussian fit also plotted as a reference. (c) Both distributions are then normalized to their mean value and standard deviation for direct comparison.

domain around mid-radius and for a time-window of approximately $5.10^{4} \omega_{c}^{-1}$ during the steady-state regime. With these intervals, the number of points available for the statistics is roughly $7.510^{4}$. As the time average of the fluxes may vary depending on the radial position, we consider for a given flux $\Gamma$ the PDF of $\Gamma-\langle\Gamma\rangle$ where $\langle\Gamma\rangle$ is the time-average of the flux at a given radial position. The resulting statistical distributions are presented in figure 3(a) for the turbulent heat flux and figure 3(b) for the turbulent Reynolds stress. In both graphs, a Gaussian fit is plotted for comparison. The two distributions exhibit similar properties, namely a strong asymmetry and a heavy tail for large positive values of the quantity. In order to compare them directly, the distributions can be normalized to their respective mean values and standard deviations, allowing them to be represented on the same graph, figure 3(c). Once normalized, the two PDFs appear remarkably similar, in particular in terms of the tails of the distributions. 
A similar result was obtained recently with the XGC1p gyrokinetic code, where the heat flux had a similar distribution as the opposite of the Reynolds stress [32]. In the latter simulation, the choice of density and temperature profile led to a turbulent drive localized near the edge of the domain, which may explain why in that case toroidal rotation was driven from the edge inward.

The characteristics of the distributions can be quantified by computing their third and fourth order moments. The normalized third central moment, or skewness, measures the asymmetry of a distribution and is exactly zero for a symmetric distribution. For the PDFs in figure 3, we find a skewness of approximately 0.79 for both fluxes. The normalized fourth central moment, or kurtosis, measures the weight of the tails in the distribution. A positive kurtosis is indicative of flat distributions, i.e. with heavy tails, while the kurtosis of a Gaussian distribution is exactly zero. We obtain values of roughly 1.7 for the heat flux and 1.5 for the Reynolds stress, clearly departing from a Gaussian in both cases. Note that the fluxes used for the analysis are flux-surface averaged, and the intermittency would be more pronounced for the local (poloidally and toroidally) fluxes, as observed experimentally [35].

In addition to the statistical analysis of the turbulent fluxes, it is interesting to investigate the difference between the dynamics of the toroidal Reynolds stress and of the time evolution of toroidal momentum (noted $\mathcal{L}_{\varphi}$ ). Although the latter is governed by the divergence of the Reynolds stress, a statistical analysis by means of PDFs reveals that they exhibit very different dynamics. The PDF of $\partial_{t} \mathcal{L}_{\varphi}$ is shown in figure 4(a), and is then normalized to its mean value and standard deviation for comparison with the turbulent Reynolds stress in figure 4(b). We recall that, although momentum transport contains several terms as presented for example in [15], the dominant term is the divergence of the turbulent toroidal Reynolds stress, with a transport equation of the form $\partial_{t} \mathcal{L}_{\varphi}+\nabla \Pi_{r, \varphi}=0$ where $\Pi_{r, \varphi}$ is the Reynolds stress.

The PDF for $\partial_{t} \mathcal{L}_{\varphi}$ is quite different from the PDF of the Reynolds stress obtained in figure $3(\mathrm{~b})$. The tails of the PDF, while still present, appear less important, as characterized by the kurtosis of the distribution of approximately 0.46 , to be compared with 1.5 for the Reynolds stress. Also the PDF of $\partial_{t} \mathcal{L}_{\varphi}$ is much more symmetric, with a skewness of approximately 0.1, which is only marginally larger than the expected precision considering the number of points in the distribution. We recall that the PDFs of heat flux and Reynolds stress had a skewness of approximately 0.79. Note that a similar result can be shown by comparing the statistics of the turbulent heat flux and of the time derivative of the local ion temperature. This suggests that, although large events are very significant in terms of heat and momentum fluxes, their effect on the profiles may not systematically be of importance. One possible interpretation of this would be that the larger events in terms of fluxes are also associated with larger radial extents, leading to moderate local modifications of the profiles.

Finally, a useful tool when analyzing the correlation between two turbulent fluxes $\Gamma_{1}$ and $\Gamma_{2}$ is the technique of joint $P D F$, which represents the statistical distribution of the variable $\left(\Gamma_{1}, \Gamma_{2}\right)$. Obviously, this technique requires larger datasets than standard 


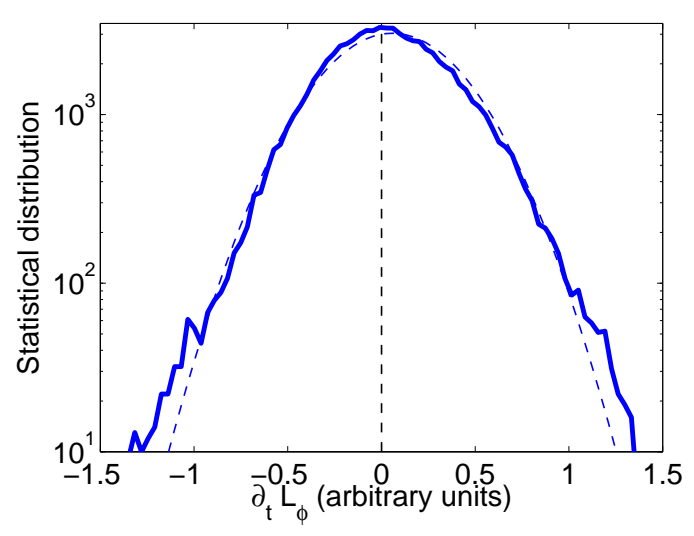

(a)

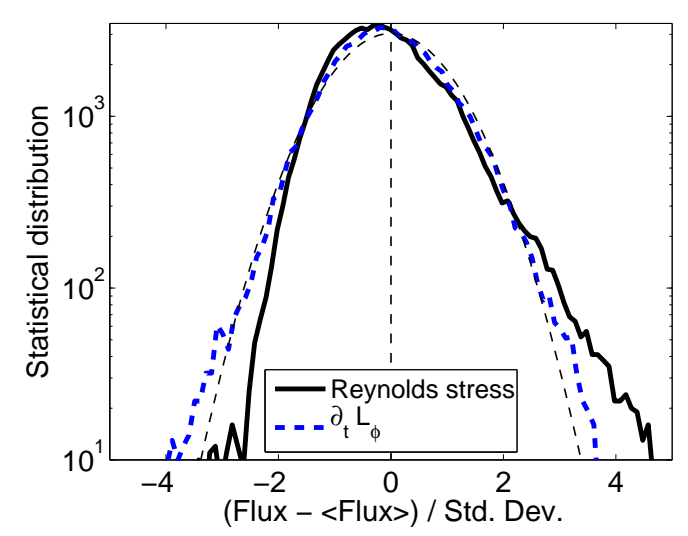

(b)

Figure 4. (a) Statistical distribution functions of the time derivative of the toroidal angular momentum $\left(\partial_{t} \mathcal{L}_{\varphi}\right)$, around mid-radius $\mathrm{r} / \mathrm{a}=0.5$, with a Gaussian fit also plotted as a reference. (c) The distribution is normalized to its mean value and standard deviation for direct comparison with the distribution of the turbulent toroidal Reynolds stress.

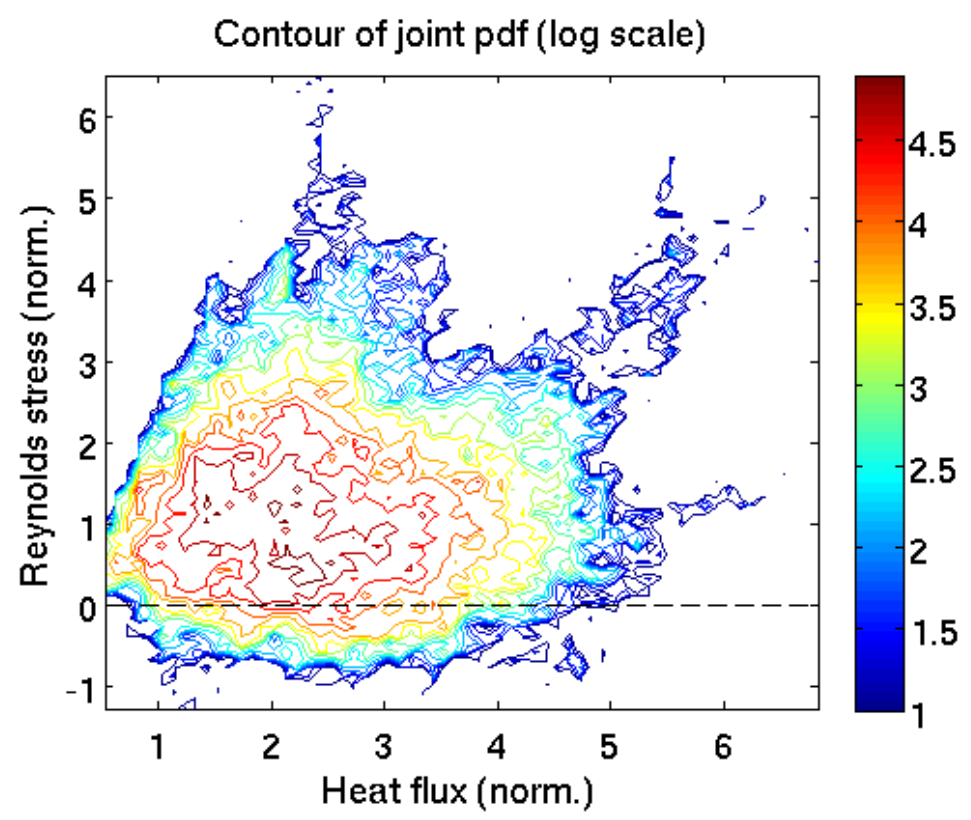

Figure 5. Logarithmic contour of the joint statistical distribution of turbulent heat flux and toroidal Reynolds stress. Both fluxes are normalized to their respective standard deviations.

PDFs, as the variable becomes two dimensional. Nevertheless, the $7.510^{4}$ points previously used are sufficient to obtain a well-resolved joint PDF of turbulent heat flux and Reynolds stress, as shown in figure 5. A significant number of events with both large turbulent Reynolds stress and heat flux is clearly identified, which can be associated with the large-scale avalanches observed in both fluxes [32]. Other events are also observed with large heat flux and weak Reynolds stress (and vice versa), suggesting 
that decorrelated bursts of either flux are also present. Finally, a striking feature of the joint PDF is the two lines which can be drawn from the origin of the figure, one corresponding to the minimum value of the Reynolds stress and another for growing heat flux and Reynolds stress. These lines are reminiscent of the cycle observed during the initial front propagation of the simulation in the previous section (figure 1(b)), suggesting that the mechanism associated with this initial front and the generation of a dipolar toroidal rotation profile may also be at work during the statistical steady-state of the simulation.

\section{Impact of scrape-off-layer flows on core toroidal rotation}

As mentioned previously, the gyrokinetic model used in GYSELA verifies a local conservation law for toroidal angular momentum, with no momentum source. Thus, the role of boundary conditions is crucial as they represent the only source of net toroidal rotation. Experimentally, there is evidence that flows from the scrape-off layer (SOL) of the plasma may influence toroidal rotation in the outer core [17, 18, 19], but this effect has yet to be confirmed by core turbulence simulations. In the simulation detailed in the previous sections, no-slip conditions (i.e. vanishing rotation) were imposed at the outer radial boundary, with an ad hoc diffusion ensuring exchange of momentum between the plasma and the edge of the simulation domain. This modeling choice, although it should not presumably affect the statistical dynamics of turbulent transport previously detailed, may have an impact on the toroidal rotation profiles eventually reached by the simulations. We investigate in the following the effect of various boundary conditions, corresponding to different types of edge plasma rotation, on core toroidal rotation in GYSELA simulations.

In order to provide boundary conditions for the GYSELA code, which does not model the open field line region, it is useful to consider first reduced models. From a simple one-dimensional model of the scrape-off layer, assuming constant temperature and no momentum source, one can obtain (see for example [36]) the steady-state profile of the parallel Mach number as

$$
\nabla_{\|} M=\frac{S}{n c_{s}} \frac{1+M^{2}}{1-M^{2}}
$$

where $S$ is the particle source, $n$ is the ion density and $c_{S}$ is the isothermal ion acoustic velocity. For a simple limiter geometry with a homogeneous particle source, one can integrate (7), leading to the (solid blue) profile of Mach number shown in figure 6(a). Notice that the Mach number goes to -1 and +1 at both ends, which correspond to the two sides of the limiter, with infinite derivatives along the magnetic field line. This basic profile can be modified when taking into account the asymmetry of the particle source or a different geometry. As an example, an output from the fluid code SOLEDGE-2D [37] in the case of a strongly ballooned source is given in figure 6(a).

Shifting to the closed field line region before investigating the question through gyrokinetic simulations, it is also possible to estimate analytically how such profiles will 


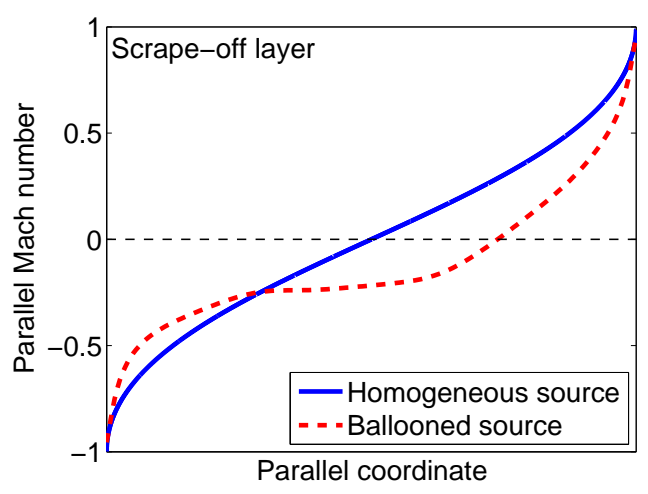

(a)

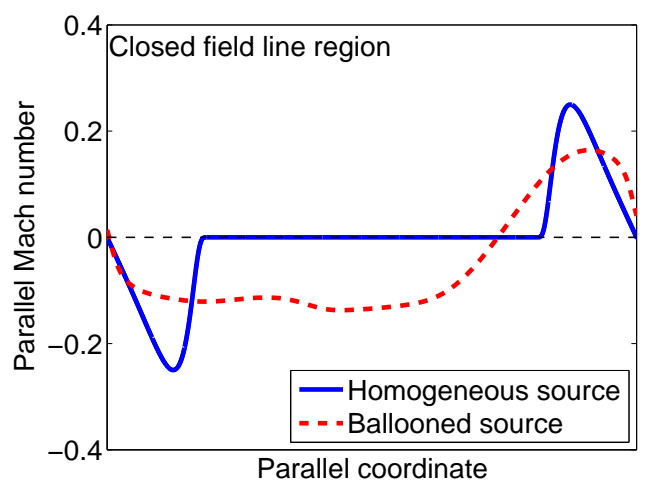

(b)

Figure 6. Simple profiles for the parallel Mach number along the magnetic field line in (a) the scrape-off layer (open magnetic field lines) and (b) in the region of closed field lines near the last closed flux surface. The solid blue lines correspond to analytical profiles in the case of a homogeneous particle source, while the dashed red lines are outputs from SOLEDGE-2D simulations with a strongly ballooned source.

penetrate the core plasma in the case of purely diffusive momentum transport. We consider a stationary two-dimensional diffusive model for density $n$ and parallel flow $\Gamma$ in the closed-field line region near the last closed magnetic flux surface (LCFS), which reads

$$
\begin{aligned}
& -D \Delta_{\perp} n+\nabla_{\|} \Gamma=0 \\
& -m_{i} \nu \Delta_{\perp} \Gamma+\nabla_{\|} \Pi=0
\end{aligned}
$$

where $D$ and $\nu$ are diffusion coefficients describing radial transport. In the limit where $M^{2} \rightarrow 0$ (i.e. low flow) and in the isothermal approximation, $\nabla_{\|} \Pi=T \nabla_{\|} n$. Thus the system of equations $(8,9)$ reduces to an equation for the parallel flow

$$
\nabla_{\|}^{2} \Gamma=\frac{D \nu}{c_{s}^{2}} \Delta_{\perp}^{2} \Gamma
$$

In Fourier space, this reads

$$
-k_{\|}^{2}=\frac{D \nu}{c_{s}^{2}} k_{\perp}^{4}
$$

Although this precise result is dependent on the choice of reduced model made here, we can expect the profiles obtained in the open field line region (figure 6(a)) to be damped in the closed field line region, with the large $k_{\|}$structures being more radially localized near the LCFS. In particular, the discontinuity between both sides of the limiter will not be observed inside the LCFS. Schematically, this leads to profiles such as figure 6(b) for the parallel Mach number. The solid blue line corresponds to the case of a symmetric source, while the dashed red line is the result of a SOLEDGE-2D simulation with a strongly ballooned source.

As a starting point for the numerical analysis, we consider a simulation with $\rho_{*}=1 / 150$ and $\nu^{*}=0.02$ which was run with conventional no-slip boundary conditions 


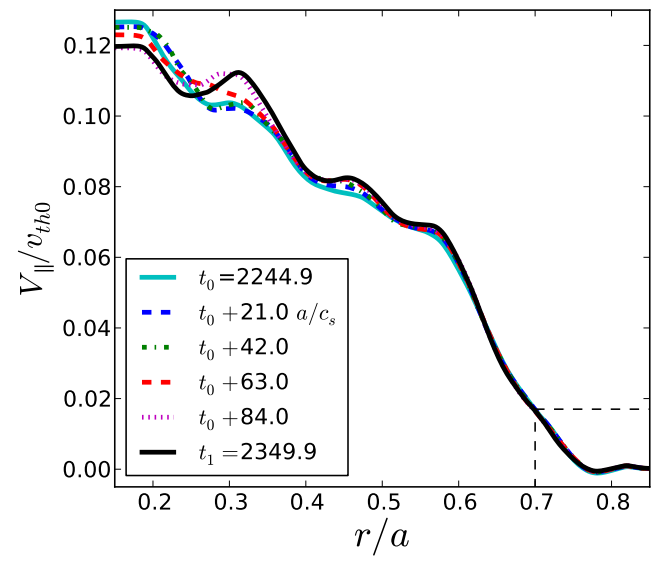

(a)

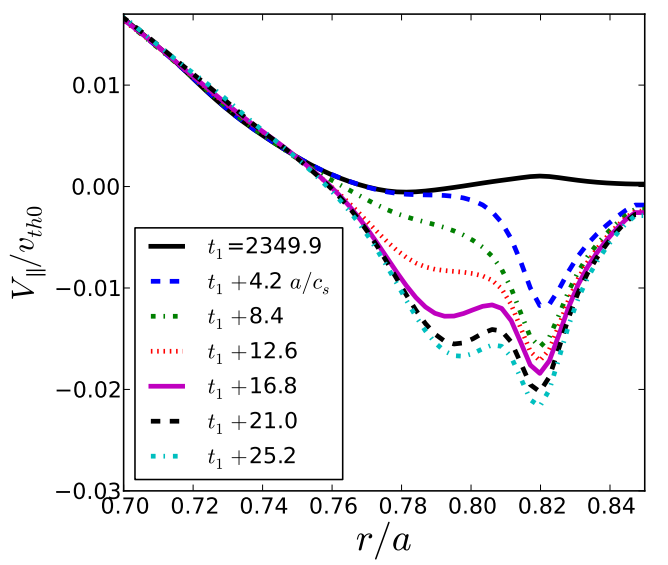

(b)

Figure 7. Radial profiles of the parallel velocity at different simulation times (normalized to $a / c_{s}$ ) showing (a) the slow evolution around a mean profile in the steady-state regime (with $\Delta t=21 a / c_{s}$ ), and (b) the rapid modification of the profile (with $\Delta t=4.2 a / c_{s}$ ) near the edge after modifying the boundary condition for parallel velocity. The velocities are normalized to a reference (i.e., constant) thermal velocity.

for approximately $3.10^{5} \omega_{c}^{-1}$. The geometry and profile shapes used were the same as previously described in section 3 but with the normalized temperature gradient $R_{0} / L_{T} \simeq 7$ in the steady-state regime, i.e. closer to the instability threshold than the simulation analyzed in the previous section. Given the input power and the pressure profile, the simulation time is comparable to the confinement time, and the plasma appears to have reached a steady-state for the mean flows, as shown in figure 7(a). From this steady-state, we impose as a boundary condition for parallel velocity a poloidally symmetric profile as presented in figure 6(b), with the poloidal position corresponding to the limiter at $\theta=0$. The maximum absolute value of the edge velocity is $V_{\text {edge }}=0.25 v_{\text {th }}$ at $\theta=\pi / 4$. As a result, the profile is rapidly modified near the edge, as can be observed in figure 7(b), and a new steady-state is reached by the flows in the simulation. Inside $r / a \simeq 0.75$, the profile does not appear to be affected by the modification of the boundary conditions, even on a long time scale. This has been confirmed by continuing the simulation with no-slip boundary conditions and comparing the obtained profiles: the difference between the two remains smaller than the level of fluctuation of the profiles around their mean steady-state values.

Considering our choice of boundary condition, it is not sufficient to analyze its effect on the core plasma in terms of flux-surface averaged flows. Even in the absence of modified boundary conditions, it is useful to recall the poloidal structure of the steadystate flows. Indeed, in the steady-state regime, the parallel velocity is not homogeneous in the poloidal direction, as can be observed in figure 8(a). Large variations of the velocity are present on a given flux-surface, essentially dominated by an $m=1$ mode, which corresponds to Pfirsch-Schlüter rotation. Note that the amplitude of this variation is of the same order of magnitude - and in fact often larger - than the flux-surface 


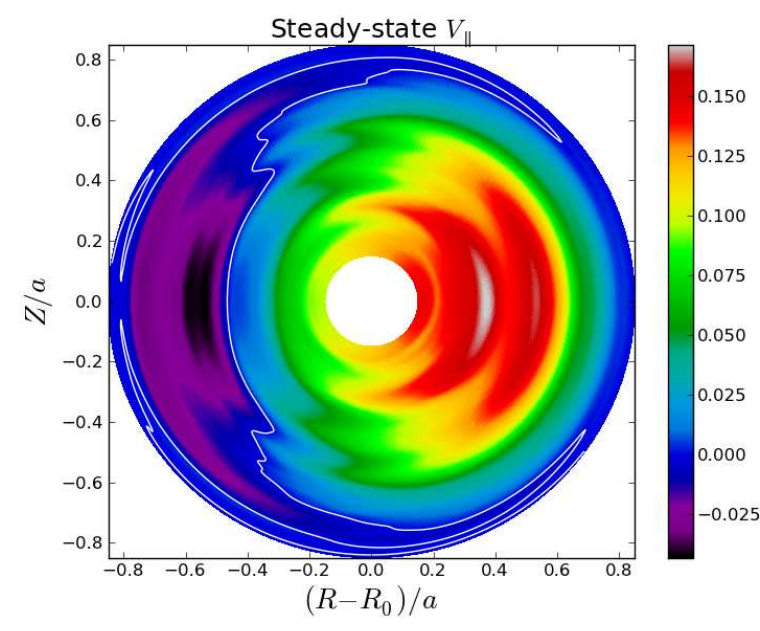

(a)

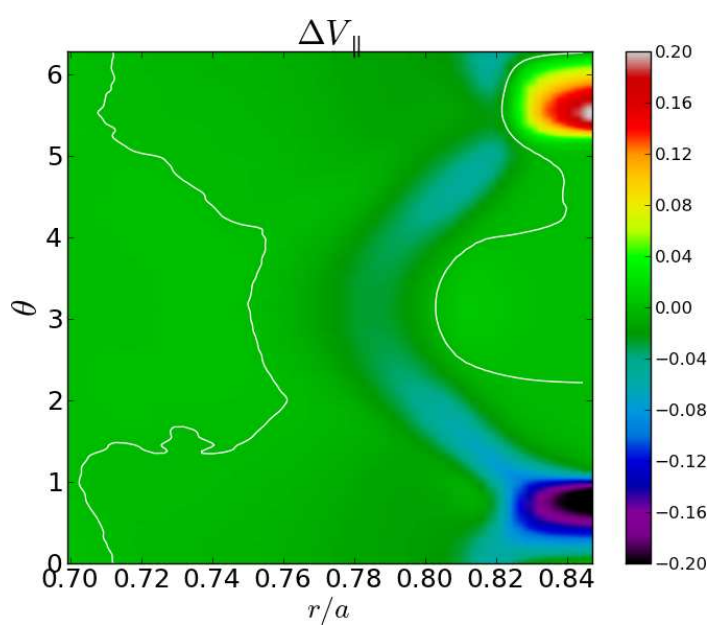

(b)

Figure 8. (a) Poloidal cross-section (averaged over $\varphi$ ) of the parallel velocity normalized to the thermal velocity - in the steady-state regime with no-slip boundary conditions. The white line corresponds to the $V_{\|}=0$ contour. (b) Modification of the parallel velocity with SOL-like boundary conditions, i.e. $\Delta V_{\|}=V_{\|}($end $)-$ $V_{\|}($steady-state), with a zoom near the outer radial boundary. The white lines correspond to $\Delta V_{\|}=0$ contours.

averaged flow, and can lead to a reversal of the sign of the parallel flow on a given fluxsurface (see the white $V_{\|}=0$ contour in figure $8(\mathrm{a})$ ). After the boundary conditions have been modified, the poloidal structure of the parallel velocity is affected, but only in a small region between $r / a \simeq 0.75$ and the outer boundary of the simulation domain, $r / a=0.85$. In order to highlight the result, the modification of the parallel velocity near the boundary, $\Delta V_{\|}=V_{\|}$(end) $-V_{\|}$(steady-state), is given in figure $8(\mathrm{~b})$. Interestingly, the effect on the poloidal structure is not localized near $\theta=0$, where the boundary condition has been modified, but rather moves to the high-field side with decreasing minor radius.

In figure 9, we analyze the transition from the last point of the domain, where the boundary condition is imposed, to the core plasma, where the flows are not affected. First of all, in the region closest to the simulation edge, the radial diffusion term damps fluctuations and governs heat and momentum transport. We recall that this radial diffusion, of the form $\frac{1}{r} \partial_{r}\left\{r D(r) \partial_{r} \bar{F}\right\}$, ensures the coupling of the plasma to the fixed temperature outside the domain and acts as a heat sink for the system in flux-driven simulations. The radial profile of the diffusion term $D(r)$ is given in figure 9 (a), along with its effect on the poloidal structure of parallel velocity, represented by the radial profile at two fixed positions of $\theta$ corresponding to the minimum and maximum value of parallel velocity. The main observation is that the boundary condition is transported as expected by the diffusion coefficient, although an asymmetry appears between positive and negative parallel rotation. The cause for this asymmetry is not understood at the moment. 


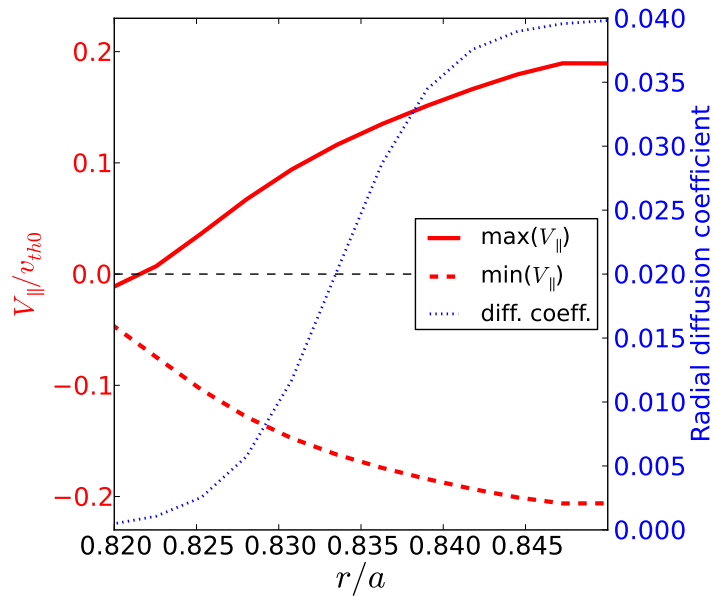

(a)

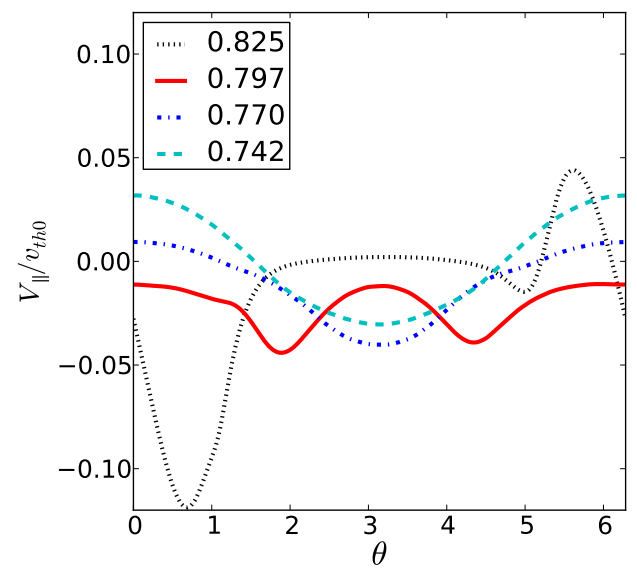

(b)

Figure 9. (a) Maximum (solid red) and minimum (dashed red) of parallel velocity in the buffer region where radial diffusion is applied, the radial profile of the diffusion coefficient is given by the dotted blue line. (b) Poloidal profiles of the parallel velocity at different radii near the boundary of the simulation domain.

In figure $9(\mathrm{~b})$, we consider the effect of the velocity outside the buffer region by analyzing the poloidal profile at several radii. At $r / a=0.825$, corresponding roughly to the limit of the buffer region, the parallel velocity still has the shape imposed at the boundary, but with an important asymmetry, as observed in figure 9(a). Further inward, we clearly observe two lobes of negative parallel velocity moving toward the high-field side, corresponding to the structures observed in figure 8. Eventually, the initial $m=1$ structure identified in figure 8 (a) is recovered for $r / a \lesssim 0.75$.

No measurable effect is observed inside $r / a \simeq 0.75$, suggesting that the impact of SOL flows on core rotation is limited to a narrow region near the edge. This key result does not appear to depend directly on the amplitude of the SOL flow, as a simulation with equivalent boundary profiles and $V_{\|} / v_{t h 0}$ doubled from 0.25 to 0.5 (at $\theta=+\pi / 4$ ) leads to a comparable penetration in the core. Additionally, running a similar simulation but with opposite boundary conditions (i.e. $V_{\|}=-0.25 v_{t h 0}$ for $\theta=-p i / 4$ ) leads to the same poloidal structure of the modified parallel velocity (figure 8) but with opposite signs for the inward propagating lobes.

The SOL flows applied as boundary conditions so far mimicked the structure of flows in the scrape-off layer, as they are observed experimentally in L-mode plasmas. When the plasma is in H-mode, the presence of a transport barrier modifies the coupling of the core plasma to the scrape-off layer. In H-mode plasmas, the boundary condition for core toroidal rotation can be described by a uniform (or "solid") rotation of relatively large amplitude at the radial position of the pedestal. Here, we investigate the effect of such boundary conditions on the plasma core, starting from the same reference simulation described previously $\left(\rho_{*}=1 / 150\right)$. Rather than an inhomogeneous poloidal profile, we set the boundary condition to a fixed non-vanishing value at the edge 


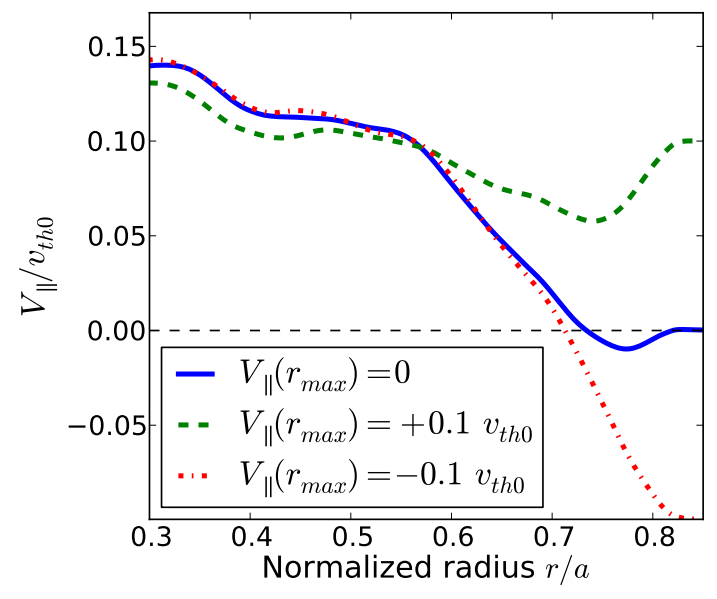

Figure 10. Mean parallel velocity profile for simulations with poloidally homogeneous boundary conditions, either no-slip or with $V_{\|}\left(r_{\max }\right)= \pm 0.1 v_{t h 0}$. The profiles have been averaged over a time frame of approximately $510^{3} \omega_{c}^{-1}$.

of the simulation domain, i.e. $r / a=0.85$. Two simulations have been performed, with $V_{\|}(r / a=0.85)= \pm 0.1 v_{t h 0}$, which is of the order of the pedestal velocities observed experimentally [38]. The resulting parallel velocity profiles are shown in figure 10 approximately $2.310^{5} \omega_{c}^{-1}$ after the modification of the boundary condition, averaged over $510^{3} \omega_{c}^{-1}$. As a reference, the simulation with no-slip conditions (i.e. $\left.V_{\|}(r / a=0.85)=0\right)$ has also been run for the same simulation time.

In contrast to the previous simulations where the effect of modified boundary conditions had a limited impact on the radial velocity profile, the homogeneous conditions applied here strongly impact the core rotation, at least up to $r / a \sim 0.6$. For the case where $V_{\|}\left(r_{\max }\right)=-0.1 v_{t h 0}$, no significant effect on parallel velocity can be measured inside this radius, as observed differences remain well within the level of fluctuations. In the case where $V_{\|}\left(r_{\max }\right)=0.1 v_{t h 0}$, the gradient of toroidal rotation is strongly reduced, and this appears to lead to a modification of the rotation profile throughout the radial domain. However, the modification observed in figure 10 for $r / a<0.6$ is only marginally larger than the observed fluctuations in the velocity profile. In order to confirm this result with more certainty, the simulation would need to be run for a time larger than the energy confinement time, which was not the case here due to limited numerical resources. Additionally, it would be useful to perform similar simulations at lower values of $\rho_{*}$ in order to investigate whether the penetration - or screening - of edge flows is dependent on the machine size. This additional step, which would allow for predictions of the impact of edge flows on core toroidal rotation of ITER, is left for future work due to the significant numerical cost of such simulations. 


\section{Conclusion}

Flux-driven simulations of turbulent heat and toroidal momentum transport have been performed with the global and full- $f$ gyrokinetic code GySELA. During the exponential growth phase of turbulence, a front of turbulent toroidal Reynolds stress propagates outward, generating a dipolar structure of toroidal rotation. As the simulation is initialized with a vanishing profile of toroidal rotation, this effect can be interpreted as the generation of intrinsic toroidal rotation by a turbulent residual stress. The Reynolds stress front is also accompanied by a front of radial heat flux, with a delay of the order of the turbulence correlation time $\tau_{c} \sim a / v_{T}$. A strong link between heat and momentum transport is also identified in the saturated turbulence regime, as avalanche-like events are observed in the turbulent fluxes, transporting both heat and momentum. For most of the avalanches in this steady-state regime, fronts of Reynolds stress are found to propagate with a delay compared to heat flux fronts. The statistical distributions of the heat flux and toroidal Reynolds stress indicate intermittent dynamics, as they are positively skewed with a heavy tail for events corresponding to large fluxes.

The conservation of angular momentum implies that scrape-off layer flows may play an important role in determining core toroidal rotation. This issue is investigated in gyrokinetic simulations of toroidal momentum transport by modifying the boundary conditions. On the one hand, imposing a simple poloidal profile mimicking edge flows in a limited L-mode plasma has only a radially localized effect on core toroidal rotation, although the penetration of such flows exhibits clear non-diffusive characteristics. On the other hand, adding an offset rotation at the edge of the simulation domain can have a strong impact on core rotation in a large fraction of the radial domain.

\section{Acknowledgments}

The authors acknowledge fruitful discussions with the participants of the sixth "Festival de Théorie" held in Aix-en-Provence in 2011, as well as helpful discussions with H. Bufferand and P. Tamain on the topic of scrape-off layer flows. This work was supported by ANR grant GYPSI ANR-10-BLAN-941. The simulations were performed with the HPC resources of CCRT and CINES under the allocation 2009052224 made by GENCI (Grand Equipement National de Calcul Intensif). This work, supported by the European Communities under the contract of Association between EURATOM and CEA, was carried out within the framework of the European Fusion Development Agreement. The views and opinions expressed herein do not necessarily reflect those of the European Commission.

\section{References}

[1] Bondeson A and Ward D J 1994 Phys. Rev. Lett. 722709

[2] Shaing K C 2001 Phys. Rev. Lett. 87245003

[3] Cole A J, Hegna C C and Callen J D 2007 Phys. Rev. Lett. 99(6) 065001 
[4] Garbet X, Abiteboul J, Trier E, Gürcan O, Sarazin Y, Smolyakov A, Allfrey S, Bourdelle C, Fenzi C, Grandgirard V, Ghendrih P and Hennequin P 2010 Phys. Plasmas 17072505

[5] Yoshida M, Koide Y, Takenaga H, Urano H, Oyama N, Kamiya K, Sakamoto Y, Kamada Y and the JT-60 Team 2006 Plasma Phys. Control. Fusion 481673

[6] de Vries P C, Salmi A, Parail V, Giroud C, Andrew Y, Biewer T M, Crombé K, Jenkins I, Johnson T, Kiptily V, Loarte A, Lönnroth J, Meigs A, Oyama N, Sartori R, Saibene G, Urano H, Zastrow K D and Contributors J E 2008 Nucl. Fusion 48035007

[7] Fenzi C, Garbet X, Trier E, Hennequin P, Bourdelle C, Aniel T, Colledani G, Devynck P, Gil C, Gürcan O D, Manenc L, Schneider M, Segui J L and the Tore Supra team 2011 Nucl. Fusion 51103038

[8] Lazzaro E, Buttery R J, Hender T C, Zanca P, Fitzpatrick R, Bigi M, Bolzonella T, Coelho R, DeBenedetti M, Nowak S et al. 2002 Phys. Plasmas 93906

[9] Garofalo A M, Burrell K H, DeBoo J C, deGrassie J S, Jackson G L, Lanctot M, Reimerdes H, Schaffer M J, Solomon W M and Strait E J 2008 Phys. Rev. Lett. 101(19) 195005

[10] Garbet X, Idomura Y, Villard L and Watanabe T H 2010 Nucl. Fusion 50043002

[11] Idomura Y, Urano H, Aiba N and Tokuda S 2009 Nucl. Fusion 49065029

[12] Sarazin Y, Grandgirard V, Abiteboul J, Allfrey S, Garbet X, Ghendrih P, Latu G, Strugarek A and Dif-Pradalier G 2010 Nucl. Fusion 50054004

[13] Görler T, Lapillonne X, Brunner S, Dannert T, Jenko F, Aghdam S K, Marcus P, McMillan B F, Merz F, Sauter O, Told D and Villard L 2011 Phys. Plasmas 18056103

[14] Scott B D and Smirnov J 2010 Phys. Plasmas 17112302

[15] Abiteboul J, Grandgirard V, Garbet X, Allfrey S J, Ghendrih P, Latu G, Sarazin Y and Strugarek A 2011 Phys. Plasmas 18082503

[16] Idomura Y 2012 Comput. Sci. Disc. 5014018

[17] LaBombard B, Rice J E, Hubbard A E, Hughes J W, Greenwald M, Irby J, Lin Y, Lipschultz B, Marmar E S, Pitcher C S et al. 2004 Nucl. Fusion 441047

[18] Gunn J P, Boucher C, Dionne M, Ďuran I, Fuchs V, Loarer T, Nanobashvili I, Pánek R, Pascal J Y, Saint-Laurent F et al. 2007 J. Nucl. Mater. 363484

[19] Hennequin P et al. 2010 37th EPS Conference on Plasma Physics, Dublin p P1.1040

[20] Grandgirard V, Sarazin Y, Angelino P, Bottino A, Crouseilles N, Darmet G, Dif-Pradalier G, Garbet X, Ghendrih P, Jolliet S et al. 2007 Plasma Phys. Control. Fusion 49 B173

[21] Grandgirard V, Brunetti M, Bertrand P, Besse N, Garbet X, Ghendrih P, Manfredi G, Sarazin Y, Sauter O, Sonnendrücker E et al. 2006 J. Comput. Phys. 217395

[22] Brizard A J and Hahm T S 2007 Rev. Mod. Phys. 79421

[23] Dif-Pradalier G, Diamond P H, Grandgirard V, Sarazin Y, Abiteboul J, Garbet X, Ghendrih P, Latu G, Strugarek A, Ku S and Chang C S 2011 Phys. Plasmas 18062309

[24] Sarazin Y, Grandgirard V, Abiteboul J, Allfrey S, Garbet X, Ghendrih P, Latu G, Strugarek A, Dif-Pradalier G, Diamond P H, Ku S, Chang C S, McMillan B F, Tran T M, Villard L, Jolliet S, Bottino A and Angelino P 2011 Nucl. Fusion 51103023

[25] Dif-Pradalier G, Diamond P H, Grandgirard V, Sarazin Y, Abiteboul J, Garbet X, Ghendrih P, Strugarek A, Ku S and Chang C S 2010 Phys. Rev. E 82025401

[26] Brizard A J and Tronko N 2011 Phys. Plasmas 18082307

[27] Peeters A G, Angioni C and Strintzi D 2007 Phys. Rev. Lett. 98265003

[28] Hahm T S, Diamond P H, Gürcan O D and Rewoldt G 2007 Phys. Plasmas 14072302

[29] Diamond P H, McDevitt C J, Gürcan O D, Hahm T S, Wang W X, Yoon E S, Holod I, Lin Z, Naulin V and Singh R 2009 Nucl. Fusion 49045002

[30] Peeters A G, Angioni C, Bortolon A, Camenen Y, Casson F J, Duval B, Fiederspiel L, Hornsby W A, Idomura Y, Hein T, Kluy N, Mantica P, Parra F I, Snodin A P, Szepesi G, Strintzi D, Tala T, Tardini G, de Vries P and Weiland J 2011 Nucl. Fusion 51094027

[31] Holod I, Lin Z and Xiao Y 2012 Phys. Plasmas 19012314

[32] Ku S, Abiteboul J, Diamond P H, Dif-Pradalier G, Kwon J M, Sarazin Y, Hahm T S, Garbet X, 
Turbulent momentum transport in core tokamak plasmas and penetration of SOL flows 20

Chang C S, Latu G, Yoon E S, Ghendrih P, Yi S, Strugarek A, Solomon W and Grandgirard V 2012 Nucl. Fusion 52063013

[33] Ku S, Chang C S and Diamond P H 2009 Nucl. Fusion 49115021

[34] Kwon J M, Yi S, Rhee T, Diamond P H, Miki K, Hahm T S, Kim J Y, Gürcan O D and McDevitt C 2012 Nucl. Fusion 52013004

[35] Nagashima Y, Itoh S I, Inagaki S, Arakawa H, Kasuya N, Fujisawa A, Kamataki K, Yamada T, Shinohara S, Oldenbürger S, Yagi M, Takase Y, Diamond P H and Itoh K 2011 Phys. Plasmas 18070701

[36] Stangeby P C 2000 The plasma boundary of magnetic fusion devices (Institute of Physics Publishing, Bristol)

[37] Isoardi L, Chiavassa G, Ciraolo G, Haldenwang P, Serre E, Ghendrih P, Sarazin Y, Schwander F and Tamain P 2010 J. Comput. Phys. 2292220

[38] Rice J E, Ince-Cushman A, deGrassie J S, Eriksson L G, Sakamoto Y, Scarabosio A, Bortolon A, Burrell K H, Duval B P, Fenzi-Bonizec C, Greenwald M J, Groebner R J, Hoang G T, Koide Y, Marmar E S, Pochelon A and Podpaly Y 2007 Nucl. Fusion 471618 